\title{
$H N F 1 b$ is involved in prostate cancer risk via modulating androgenic hormone effects and coordination with other genes
}

\author{
Y.L. Hu ${ }^{1 *}$, D. Zhong ${ }^{2 *}$, F. Pang ${ }^{3}$, Q.Y. Ning ${ }^{2}$, Y.Y. Zhang ${ }^{1}$, G. Li ${ }^{1}$, J.Z. Wu ${ }^{2}$ \\ and Z.N. $\mathrm{Mo}^{4}$ \\ ${ }^{1}$ Medical Scientific Research Centre, Guangxi Medical University, Nanning, \\ Guangxi Zhuang Autonomous Region, China \\ ${ }^{2}$ Department of Infectious Diseases, \\ The First Affiliated Hospital of Guangxi Medical University, Nanning, \\ Guangxi Zhuang Autonomous Region, China \\ ${ }^{3}$ The First Clinic College, Guangxi Medical University, Nanning, \\ Guangxi Zhuang Autonomous Region, China \\ ${ }^{4}$ Department of Urology, \\ The First Affiliated Hospital of Guangxi Medical University, Nanning, \\ Guangxi Zhuang Autonomous Region, China \\ *These authors contributed equally to this study. \\ Corresponding authors: J.Z. Wu / Z.N. Mo \\ E-mail:jzwu_gxmu@163.com / mozengnan@gmail.com
}

Genet. Mol. Res. 12 (2): 1327-1335 (2013)

Received July 16, 2012

Accepted January 22, 2013

Published April 25, 2013

DOI http://dx.doi.org/10.4238/2013.April.25.4

\begin{abstract}
Prostate cancer is one of the most commonly diagnosed male malignancies. Genome wide association studies have revealed $H N F 1 b$ to be a major risk gene for prostate cancer susceptibility. We examined the mechanisms of involvement of HNF1b in prostate cancer development. We integrated data from Gene Expression Omnibus prostate cancer genes from the Dragon Database of Genes Implicated in Prostate Cancer, and used meta-analysis data to generate a panel of $H N F 1 b$-associated prostate cancer risk genes. An RT-PCR was used to assess expression levels in DU145, PC3, LNCaP, and
\end{abstract}


RWEP-1 cells. Twelve genes (BAG1, DDR1, ERBB4, ESR1, HSPD1, $I G F B P 2, I G F B P 5, N R 4 A 1, P A W R, P I K 3 C G, R A P 2 A$, and TPD52) were found to be associated with both $H N F 1 b$ and prostate cancer risk. Six of them (BAG1, ERBB4, ESR1, HSPD1, NR4A1, and PIK3CG) were mapped to the KEGG pathway, and submitted to further gene expression assessment. $H N F 1 b, N R 4 A 1$, and $H S P D 1$ were found to be highly expressed in the LNCaP androgenic hormone-dependent cell line. Compared to expression levels in wild-type prostate cancer cells, NR4A1, HSPD1, ERBB4, and ESR1 expression levels were also found to be significantly increased in the $H N F 1 b$-transfected cells. We conclude that the mechanism of action of $H N F 1 b$ in prostate cancer involves modulation of the association between androgenic hormone and prostate cancer cells. Gene-gene interaction and coordination should be taken into account to determine relationships between specific loci and diseases.

Key words: Prostate cancer; HNF1b; Androgenic hormone; Mechanism

\section{INTRODUCTION}

Prostate cancer is one of the most commonly diagnosed male malignancies, with the third leading morbidity rate and the sixth mortality rate in male cancer worldwide (Denmeade and Isaacs, 2004). However, the etiology of prostate cancer remains obscure. Genome-wide association (GWA) studies, characterized by high-throughput results, are useful for suggesting risk candidate genes associated with common diseases (Manolio et al., 2008). Several recent GWA studies (Gudmundsson et al., 2007; Eeles et al., 2008; Thomas et al., 2008; Takata et al., 2010; Liu et al., 2011), which are based on different ethnicities including European, Chinese, and Japanese, have universally identified hepatic nuclear factor 1 beta $(H N F 1 b)$ as a risk locus for prostate cancer. This accumulating evidence has suggested a high-risk relationship between $H N F 1 b$ and prostate cancer susceptibility. Despite that GWA studies have made it easy to obtain substantial information about genetic variants contributing to cancer susceptibility, they have been unable to elucidate the underlying mechanisms of the diseases or to guarantee the biological significance of the findings (Min et al., 2012).

$H N F 1 b$ is located on $17 \mathrm{q} 21.3$ and is responsible for encoding a number of members of the homeodomain-containing superfamily of transcription factors (Tronche and Yaniv, 1992). $H N F 1 b$ is well known to be involved in maturity-onset diabetes in the young, renal disease, and embryonic survival (Kato and Motoyama, 2009; Chen et al., 2010), but the tumorigenesis mechanism of $H N F 1 b$ in prostate cancer is not yet clearly elucidated. In an attempt to provide a preliminary insight into this field, we hypothesized that a certain set of genes is expressed in a manner associated or coordinated with $H N F 1 b$ in carcinogenesis. To address the hypothesis, we applied the microarray datasets from Gene Expression Omnibus (GEO) to generate genes whose expression is significantly correlated with $H N F 1 b$. The $H N F 1 b$-correlated genes were then compared with genes reported to be implicated in prostate cancer by Dragon Database (DDPC; http://apps.sanbi.ac.za/ddpc/) and our previous meta-analysis of 6 independent gene expression profiling studies about prostate cancer from GEO (Ning et al., 2011). Thus, $H N F 1 b$-associated prostate cancer genes were obtained. To further establish a functional mo- 
lecular background of $H N F 1 b$, we selected genes that could be mapped to any explicit Kyoto Encyclopedia of Genes and Genomes (KEGG) pathway to evaluate the expression level by quantitative RT-PCR in 3 prostate cancer cell lines and 1 normal prostate epithelial cell line.

\section{MATERIAL AND METHODS}

\section{GEO database}

The GEO database (http://www.ncbi.nlm.nih.gov/geo/) is a public archive for highthroughput functional genomic datasets (Wilhite and Barrett, 2012). We searched GEO with the key word " $H N F 1 B$ " and included data that met the following criteria: 1) the data were about gene expression in wild-type and induced $H N F 1 B$ cell lines; 2) organisms were humans or rats; 3 ) the data provided complete microarray raw or normalized data. Finally, we found 2 datasets that met the inclusion criteria, with one for humans (GDS 1499 record) and the other one for Rattus norvegicus (GDS 905 record). To obtain the co-expressed genes of HNF1b, we downloaded text files of the included datasets by GEO accession. Using the gene ID conversion tool in Database for Annotation, Visualization, and Integrated Discovery (http://david.abcc.ncifcrf.gov/), we explored the official gene names in batch for the ID references in the GEO downloaded files (Huang et al., 2009). The gene expression data of $H N F 1 b$-induced and -noninduced cells were compared using the Student $t$-test. The data of humans and rats were calculated separately. This file was assumed as the basis of our further comparison.

\section{Genes implicated in DDPC}

DDPC serves as a comprehensive repository that integrates data about prostate cancer from other biological research areas including molecular interactions, pathways, gene ontologies, and gene regulation at the molecular level (Maqungo et al., 2011). All the genes implicated in prostate cancer by DDPC were downloaded.

Finally, 3 groups of data, including $H N F 1 b$ co-expression genes from GEO, prostate cancer genes from DDPC, and meta-analysis of 6 independent gene expression profiling studies about prostate cancer (Ning et al., 2011), were checked manually to identify genes that were themselves not only related to $H N F 1 b$ but also associated with prostate cancer susceptibility.

\section{KEGG pathways}

KEGG data are widely used in pathway analysis. KEGG serves as a public archive of manually drawn pathway maps to present comprehensive and systemic interpretation for biological functions of genes (Wixon and Kell, 2000). Comprehensive searches were conducted in KEGG to select genes involved in the pathways for further analysis.

\section{Cell lines and culture}

The LNCaP cell line was kindly provided by the Institute of Molecular Biology, Nan- 
Kai University, China. The DU145 cell line was purchased from the Wuhan Boster Bio-Engineering Limited Company, China. The PC3 and RWPE-1 cell lines were purchased from the XiangYa Central Experiment Laboratory, Central South University, China. DU145 and LNCap were cultured in RPMI 1640 medium (Hyclone Company, USA) supplemented with $10 \%$ fetal bovine serum (FBS; Hyclone). PC3 were cultured in DMEM/F12 (Hyclone) supplemented with 7\% FBS. RWPE-1 cells were cultured in Defined Keratinocyte-SFM (Invitrogen, USA) in the absence of FBS. All media were supplemented with $50 \mathrm{U} / \mathrm{mL}$ penicillin and 50 $\mu \mathrm{g} / \mathrm{mL}$ streptomycin (Solarbio, China). The cells were maintained at $37^{\circ} \mathrm{C}$ in $5 \% \mathrm{CO}_{2}$ in a sterile incubator. An $H N F$-b-transfected PC3 cell line was also established to assess the changes in gene expression.

\section{Plasmid construction and transfection of target cells}

The plasmid vectors for $H N F 1 b$ were purchased from GenePharma Co., Ltd. (Shanghai, China). The transfection was conducted according to the user manual. Briefly, PC3 cells were seeded on 24-well culture plates at a density of $0.5 \times 10^{5}$ cells/well $24 \mathrm{~h}$ prior to transfection. Cells at $80 \%$ confluence were then transfected with vectors (U6/GFP/Neo$H N F 1 b$-1-homo-1759) and incubated for $48 \mathrm{~h}$ in serum-free medium. The green fluorescence protein was used to detect cell transfection efficiency.

\section{Gene expression analysis}

The analysis of every gene was carried out in triplicate each time, and the experiments were replicated twice with cell cultures. Glyceraldehyde-3-phosphate dehydrogenase was used to normalize target gene expression. Total RNA was extracted and reverse transcribed to cDNA following the manufacturer protocol using the RNeasy kit (Qiagen, Germany) and cDNA Synthesis kit (Fermentas, Canada), respectively. The reaction mixture contained $10 \mu \mathrm{L}$ FastStart Universal SYBR Green Master (Roche, Germany), $6 \mu \mathrm{M}$ forward primers, $6 \mu \mathrm{M}$ reverse primers, and $10 \mathrm{ng}$ cDNA, with RNAase-free water added to a total volume of $20 \mu \mathrm{L}$. The amplification and real-time analysis were run for 40 cycles with the following parameters: $95^{\circ} \mathrm{C}$ for $10 \mathrm{~min}$ for activation of FastStart Taq DNA polymerase; $60^{\circ} \mathrm{C}$ for $1 \mathrm{~min}$ for amplification and real-time analysis. The expression levels of the genes studied were determined using $2^{-\Delta \Delta C T}$.

$\Delta \Delta \mathrm{CT}=\Delta \mathrm{CT}^{\text {cancer/transfected cell lines }}-\Delta \mathrm{CT}^{\text {normal cell lines }}$, and $\Delta \mathrm{CT}=\Delta \mathrm{CT}$ (target gene) $\Delta \mathrm{CT}$ (endogenous reference) (Livak and Schmittgen, 2001). The primers are listed in Table 1.

\begin{tabular}{|c|c|c|c|}
\hline Genes & Official full name & Forward primer $\left(5^{\prime} \rightarrow 3^{\prime}\right)$ & Reverse primer $\left(5^{\prime} \rightarrow 3^{\prime}\right)$ \\
\hline$\overline{G A P D H}$ & Glyceraldehyde-3-phosphate dehydrogenase & agaaggctggggctcatttg & aggggccatccacagtcttc \\
\hline$H N F 1 b$ & Hepatic nuclear factor 1 beta & cagactcacagcetgaaccc & ggtgactagttgttgaggagga \\
\hline$B A G 1$ & BCL2-associated athanogene & aagaacagtccacaggaagagg & cagtgtgtcaatctcctccaag \\
\hline ERBB 4 & v-erb-a erythroblastic leukemia viral oncogene homolog 4 & ccgaggatgagtatgtgaatga & ggttccagtagtcagggttgtc \\
\hline ESR 1 & Estrogen receptor 1 & tcctgatgattggtctcgtct & acattttccetggttcetgtc \\
\hline HSPDI & Heat shock $60 \mathrm{KDa}$ protein 1 (chaperonin) & tgtttggagaagagggattga & gagecttgtcaccttttcctt \\
\hline NR4Al & Nuclear receptor subfamily 4 , group A, member 1 & ctgecaatctcctcacttcc & cggagagcaggtcgtagaac \\
\hline$P I K 3 C G$ & Phosphoinostide-3 kinase, catalytic, gamma polypeptide & gaataggcgacagacacaatga & aaagaggaagtcaggggttagc \\
\hline
\end{tabular}




\section{Statistical analysis}

Data are reported as means $\pm \mathrm{SE}$ (standard error of the mean). The analyses among the 4 cell lines were performed by ANOVA and the post hoc Bonferroni/Dunn test. The comparisons between the wild-type PC 3 and $H N F 1 b$-transfected PC 3 were performed by the Student $t$-test. All $\mathrm{P}$ values were two-sided, and $\mathrm{P}<0.05$ was considered to be significant. Relative expression of target genes was considered to be upregulation if the value $\geq 1.5$, or to be undetermined if gene expression could not be determined (Das et al., 2010).

\section{RESULTS}

\section{Genes related with $H N F 1 b$}

A total 1019 genes showed significant differential expression in humans (GDS 1499 record) by comparing gene expression data of the wild-type and HNF1b-induced HEK293 cell line. A total of 1243 genes (GDS 905 record) showed significant differential expression in $R$. norvegicus by comparing the gene expression data of the wild-type and HNF1b-induced INS-1 cell line (Table S1).

\section{Common prostate cancer genes in DDPC and meta-analysis}

A total of 563 genes associated with prostate cancer were downloaded from DDPC. A total of 176 common genes were identified between the downloaded data and the findings of our previous meta-analysis (Table S2). Herein, we defined them as DDPC-meta prostate cancer risk genes.

\section{Genes associated with $H N F 1 b$ and prostate cancer}

With the software available at http://jura.wi.mit.edu/bioc/tools/compare.php, we compared $H N F 1 b$ genes and prostate cancer risk genes. No intersection was observed between the gene data of rat and 176 DDPC-meta genes, while 12 genes were found to be identical between the human genes and 176 DDPC-meta genes. They were BAG1, DDR1, ERBB4, ESR1, HSPD1, IGFBP2, IGFBP5, NR4A1, PAWR, PIK3CG, RAP2A, and TPD52. Specifically, 6 genes $(B A G 1, E R B B 4, E S R 1, H S P D 1, N R 4 A 1$, and $P I K 3 C G)$, which are mapped in KEGG pathway annotations, were picked out for further assessment of mRNA levels. The details of the 6 genes, including the name, GenBank accession number, and KEGG pathway description are provided in Table 2 .

\section{Expression of selected genes in the cell lines and transfected cells}

The fold-changes of the genes in the 4 cell lines and the $H N F 1 b$-transfected PC 3 cells are shown in Table 3. Although the fold-changes varied widely between PC3, DU145, and LNCaP, an upregulated expression trend of the 7 genes was observed in the prostate cancer cell lines. The increased fold-changes were particularly obvious in the hormone-dependent LNCaP cell line. Compared to the wild-type PC3, the gene expression levels mostly showed 
significant upregulation after $H N F 1 b$ transfection, particularly in the 4 genes $N R 4 A 1, H S P D 1$, ERBB4, and ESR1 (Table 3).

\begin{tabular}{|c|c|c|c|}
\hline$\overline{\text { Genes }}$ & GenBank accession No. & KEGG ID & KEGG pathway \\
\hline$\overline{B A G 1}$ & 537 & Hsa04141 & Protein processing in endoplasmic reticulum \\
\hline \multirow{3}{*}{ ERBB 4} & 2066 & Hsa04012 & ErbB signaling pathway \\
\hline & & Hsa04020 & Calcium signaling pathway \\
\hline & & Hsa04144 & Endocytosis \\
\hline ESRI & 2099 & Has04961 & Endocrine and other factor-regulated calcium reabsorption \\
\hline \multirow[t]{2}{*}{ HSPDI } & 3329 & Hsa03018 & RNA degradation \\
\hline & & Hsa04940 & Type 1 diabetes mellitus \\
\hline NR4AI & 3164 & Hsa04010 & MAPK signaling pathway \\
\hline \multirow[t]{5}{*}{ PIK3CG } & 5294 & Hsa04012 & ErbB signaling pathway \\
\hline & & Hsa04062 & Chemokine signaling pathway \\
\hline & & Hsa04370 & VEGF signaling pathway \\
\hline & & Hsa04510 & Focal adhesion \\
\hline & & Hsa04620 & Toll-like receptor signaling pathway \\
\hline
\end{tabular}

Table 3. Expression level of the genes associated with $H N F 1 b$ in cell lines.
\begin{tabular}{lccccc}
\hline Genes & PC3 & DU145 & LNCaP & RWPE- & $H N F 1 b$-transfected PC3 \\
\hline$H N F 1 b$ & $5.56 \pm 0.61^{*}$ & $1.32 \pm 0.59$ & $18.91 \pm 0.72^{*}$ & $0.77 \pm 0.14$ & $7.37 \pm 0.98^{* *}$ \\
$B A G 1$ & $3.34 \pm 1.27$ & $1.93 \pm 0.36^{*}$ & $3.67 \pm 1.66$ & $2.23 \pm 0.11$ & $2.57 \pm 0.96^{* *}$ \\
$E R B B 4$ & $1.12 \pm 0.26$ & $1.89 \pm 0.43$ & $0.55 \pm 0.24$ & $0.83 \pm 0.31$ & $5.18 \pm 2.07^{* *}$ \\
$E S R 1$ & $4.82 \pm 1.76^{*}$ & $\mathrm{ND}$ & $\mathrm{ND}$ & $0.83 \pm 0.29$ & $8.35 \pm 2.23^{* *}$ \\
$H S P D 1$ & $14.38 \pm 3.2$ & $1.07 \pm 0.15$ & $27.4 \pm 2.15^{*}$ & $1.95 \pm 0.16$ & $39.65 \pm 7.43^{* *}$ \\
NR4A1 & $1.83 \pm 0.83$ & $\mathrm{ND}$ & $8.71 \pm 3.07^{*}$ & $2.03 \pm 0.32$ & $8.09 \pm 2.39^{* *}$ \\
$P I K 3 C G$ & $\mathrm{ND}$ & $\mathrm{ND}$ & $\mathrm{ND}$ & $\mathrm{ND}$ & $0.18 \pm 0.04^{* *}$ \\
\hline
\end{tabular}

$\mathrm{ND}=$ gene expression level was not determinate. ${ }^{* *}$ Comparisons of the wild-type PC 3 and the $H N F 1 b$-transfected PC3 $(\mathrm{P}<0.05)$. * Comparison of the four cell lines $(\mathrm{P}<0.05)$.

\section{DISCUSSION}

With the integration of microarray data in GEO and the prostate cancer risk genes in DDPC, as well as the findings of our previous meta-analysis, 12 prostate cancer risk genes (BAG1, DDR1, ERBB4, ESR1, HSPD1, IGFBP2, IGFBP5, NR4A1, PAWR, PIK3CG, RAP2A, and TPD52) were identified potentially connected and related to HNF1b. Six of them (BAG1, ERBB 4, ESR1, HSPD1, NR4A1, and PIK3CG) were found to participate in the KEGG pathways.

It was suggested that these genes may express in a manner correlated with $H N F 1 b$. The investigation for a panel of co-expressed genes is more likely to provide substantial information for complicated diseases than a single gene analysis. Thus, we evaluated the expression levels of $H N F 1 b$ and the 6 genes using quantitative real-time PCR. These 7 genes were more or less found overexpressed in the prostate cancer cell lines. Meanwhile, the 6 potentially $H N F 1 b$-associated genes were mostly found upregulated in the $H N F 1 b$-transfected cells. We speculated that the main function of individual gene and genetically synergistic interactions could be the underlying molecular background of diseases. Growing evidence has demonstrated the carcinogenic character of HNF1b in a number of cancers, including those of the endometrium, ovary, kidney, breast, and prostate (Terasawa et al., 2006; Tommasi et al., 2009; Harries et al., 2010; Szponar et al., 2011; Setiawan et al., 2012). To the best of our 
knowledge, the underlying mechanism of $H N F 1 b$ for prostate cancer has still been poorly investigated. It is noteworthy that the fold-changes of $H N F 1 b$ expression were remarkably higher in LNCaP, an androgen-sensitive cell line, than in the other cell lines. HNF1b encodes a transcription factor in humans. Song et al. (1998) reported that in rat liver, the binding of $H N F 1$ factor with the promoter in the dehydroepiandrosterone sulfotransferase (Std) gene enables transcriptional activation of Std, which could repress androgenic steroids by catalyzing sulfonation. The Std gene is assumed not to be tissue-specific, since it was also found to modulate androgen sensitivity in a prostate cancer cell line (Chan et al., 1998). These lines of evidence strongly suggested that $H N F 1 b$ is likely to be involved in prostate cancer in a manner associated with steroid hormone metabolism.

In addition, the expression of NR4A1 and HSPDI in prostate cancer may be closely connected with $H N F 1 b$ as reflected by the finding that their expression fold-changes were significantly increased following transfection with $H N F 1 b$. NR4Al belongs to the steroid-thyroid hormone-retinoid receptor superfamily. It has a close association with steroid hormone metabolism, such as the regulation of the hypothalamo-pituitary-adrenal axis, and steroidogenesis (Hamid et al., 2008). Androgens can cause a rapid induction of NR4A1 in human LNCaP prostate cancer cells (Chang et al., 1993). Furthermore, NR4A1 plays a pivotal role in the pathway. Mitogen-activated protein kinase is a crucial signal transduction pathway associated with cancer development and controls a variety of physiological activities of cells including proliferation, differentiation, and anti-apoptosis (Johnson and Lapadat, 2002). Uemura and Chang (1998) demonstrated that NR4AI could reduce prostate cancer cell apoptosis and predicted its potential carcinogenesis role in prostate cancer.

$H S P D 1$, which has long been recognized as an evolutionarily conserved stress response chaperone, has been observed to be upregulated in prostate cancer patients (Cappello et al., 2003; Skvortsov et al., 2011). The overexpression of HSPD1 may be potentially associated with the progression and prognosis of prostate cancer (Cornford et al., 2000). HSPD1 could suppress tumor cell apoptosis by maintaining stability of mitochondria (Ghosh et al., 2010), restraining $p 53$ and enhancing a common cancer gene called survivin (Ghosh et al., 2008; Kelly et al., 2011). HSPDI has also been demonstrated to be associated with early onset of hormone refractory disease in advanced prostate cancer patients under androgen ablation therapy (Castilla et al., 2010). Taken together, the possible explanation for the prostate cancer risk role of $H N F 1 b$ could possibly be associated with modulating the relationships between androgenic hormone and prostate cancer. However, the exact mechanisms still remain to be elucidated in future studies.

The expression fold-changes of the other 4 genes (ERBB4,ESR1, BAG1, and PIK3CG) showed a coordinated association with $H N F 1 b$, although the changes of $B A G 1$ and PIK3CG were not as obvious as with ERBB4 and ESR1. They are mapped to several important signal pathways, such as vascular endothelial growth factor signaling pathway, focal adhesion, and calcium signaling pathway. The cancer susceptibility genes with mild or weak individual effects can be aggregated into a significant risk influence (Pierce and Ahsan, 2010). Future studies are in great demand to investigate how these genes contribute to tumorigenesis as a network or through synergistic effects.

There were some limitations in this study. First, 6 genes in the absence of KEGG pathways were not examined with respect to gene expression levels, and we might have missed meaningful loci. Future studies should examine these genes. Second, our data were mainly derived from prostate cell lines, and therefore, studies are needed to extensively explore the tissue 
to obtain in-depth conclusions. There were some strengths in our study. We provide a preliminary insight into the underlying mechanism of $H N F 1 b$ prostate cancer risk by studying a gene network rather than a single gene, which may help us in interpreting a disease comprehensively.

\section{CONCLUSION}

Our study implies that the role of $H N F 1 b$ in prostate cancer risk may be associated with androgenic hormone. Gene-to-gene interaction and coordination should be taken into account to fully explain the relationships between a locus of interest and diseases.

\section{ACKNOWLEDGMENTS}

Research supported by the National Natural Science Foundation of China (\#81060213 and \#81272853) and the GuangXi Nature Science Foundation (\#2011GXNSFB018100 and \#2012GXNSFAA053152).

\section{Supplementary material}

\section{REFERENCES}

Cappello F, Rappa F, David S, Anzalone R, et al. (2003). Immunohistochemical evaluation of PCNA, p53, HSP60, HSP10 and MUC-2 presence and expression in prostate carcinogenesis. Anticancer Res. 23: 1325-1331.

Castilla C, Congregado B, Conde JM, Medina R, et al. (2010). Immunohistochemical expression of Hsp60 correlates with tumor progression and hormone resistance in prostate cancer. Urology 76: 1017.e1-6.

Chan J, Song CS, Matusik RJ, Chatterjee B, et al. (1998). Inhibition of androgen action by dehydroepiandrosterone sulfotransferase transfected in PC3 prostate cancer cells. Chem. Biol. Interact. 109: 267-278.

Chang C, Saltzman A, Lee HJ, Uemura H, et al. (1993). Genomic structure, chromosomal localization and expression of an androgen inducible TR3 orphan receptor: a member of the steroid receptor superfamily. Endocrine J. 1: 541-549.

Chen YZ, Gao Q, Zhao XZ, Chen YZ, et al. (2010). Systematic review of TCF2 anomalies in renal cysts and diabetes syndrome/maturity onset diabetes of the young type 5. Chin. Med. J. 123: 3326-3333.

Cornford PA, Dodson AR, Parsons KF, Desmond AD, et al. (2000). Heat shock protein expression independently predicts clinical outcome in prostate cancer. Cancer Res. 60: 7099-7105.

Das K, Lorena PD, Ng LK, Lim D, et al. (2010). Differential expression of steroid 5alpha-reductase isozymes and association with disease severity and angiogenic genes predict their biological role in prostate cancer. Endocr. Relat. Cancer 17: 757-770.

Denmeade SR and Isaacs JT (2004). Development of prostate cancer treatment: the good news. Prostate 58: 211-224.

Eeles RA, Kote-Jarai Z, Giles GG, Olama AA, et al. (2008). Multiple newly identified loci associated with prostate cancer susceptibility. Nat. Genet. 40: 316-321.

Ghosh JC, Dohi T, Kang BH and Altieri DC (2008). Hsp60 regulation of tumor cell apoptosis. J. Biol. Chem. 283: 51885194.

Ghosh JC, Siegelin MD, Dohi T and Altieri DC (2010). Heat shock protein 60 regulation of the mitochondrial permeability transition pore in tumor cells. Cancer Res. 70: 8988-8993.

Gudmundsson J, Sulem P, Steinthorsdottir V, Bergthorsson JT, et al. (2007). Two variants on chromosome 17 confer prostate cancer risk, and the one in TCF2 protects against type 2 diabetes. Nat. Genet. 39: 977-983.

Hamid T, Malik MT, Millar RP and Kakar SS (2008). Protein kinase A serves as a primary pathway in activation of Nur77 expression by gonadotropin-releasing hormone in the LbetaT2 mouse pituitary gonadotroph tumor cell line. Int. J. Oncol. 33: 1055-1064.

Harries LW, Perry JR, McCullagh P and Crundwell M (2010). Alterations in LMTK2, MSMB and HNF1B gene expression are associated with the development of prostate cancer. BMC Cancer 10: 315.

Huang da W, Sherman BT and Lempicki RA (2009). Systematic and integrative analysis of large gene lists using DAVID bioinformatics resources. Nat. Protoc. 4: 44-57. 
Johnson GL and Lapadat R (2002). Mitogen-activated protein kinase pathways mediated by ERK, JNK, and p38 protein kinases. Science 298: 1911-1912.

Kato N and Motoyama T (2009). Hepatocyte nuclear factor-1 beta(HNF-1 beta) in human urogenital organs: its expression and role in embryogenesis and tumorigenesis. Histol. Histopathol. 24: 1479-1486.

Kelly RJ, Lopez-Chavez A, Citrin D, Janik JE, et al. (2011). Impacting tumor cell-fate by targeting the inhibitor of apoptosis protein survivin. Mol. Cancer 10: 35 .

Liu F, Hsing AW, Wang X, Shao Q, et al. (2011). Systematic confirmation study of reported prostate cancer risk-associated single nucleotide polymorphisms in Chinese men. Cancer Sci. 102: 1916-1920.

Livak KJ and Schmittgen TD (2001). Analysis of relative gene expression data using real-time quantitative PCR and the 2(-Delta Delta C(T)) Method. Methods 25: 402-408.

Manolio TA, Brooks LD and Collins FS (2008). A HapMap harvest of insights into the genetics of common disease. $J$. Clin. Invest. 118: 1590-1605.

Maqungo M, Kaur M, Kwofie SK, Radovanovic A, et al. (2011). DDPC: Dragon Database of Genes associated with Prostate Cancer. Nucleic Acids Res. 39: D980-D985.

Min JL, Nicholson G, Halgrimsdottir I, Almstrup K, et al. (2012). Coexpression network analysis in abdominal and gluteal adipose tissue reveals regulatory genetic loci for metabolic syndrome and related phenotypes. PLoS Genet. 8: e1002505.

Ning QY, Wu JZ, Zang N, Liang J, et al. (2011). Key pathways involved in prostate cancer based on gene set enrichment analysis and meta analysis. Genet. Mol. Res. 10: 3856-3887.

Pierce BL and Ahsan H (2010). Genetic susceptibility to type 2 diabetes is associated with reduced prostate cancer risk. Hum. Hered. 69: 193-201.

Setiawan VW, Haessler J, Schumacher F, Cote ML, et al. (2012). HNF1B and endometrial cancer risk: results from the PAGE study. PLoS One 7: e30390.

Skvortsov S, Schafer G, Stasyk T, Fuchsberger C, et al. (2011). Proteomics profiling of microdissected low- and highgrade prostate tumors identifies Lamin A as a discriminatory biomarker. J. Proteome. Res. 10: 259-268.

Song CS, Jung MH, Kim SC, Hassan T, et al. (1998). Tissue-specific and androgen-repressible regulation of the rat dehydroepiandrosterone sulfotransferase gene promoter. J. Biol. Chem. 273: 21856-21866.

Szponar A, Yusenko MV, Kuiper R, van Kessel AG, et al. (2011). Genomic profiling of papillary renal cell tumours identifies small regions of DNA alterations: a possible role of HNF1B in tumour development. Histopathology 58: 934-943.

Takata R, Akamatsu S, Kubo M, Takahashi A, et al. (2010). Genome-wide association study identifies five new susceptibility loci for prostate cancer in the Japanese population. Nat. Genet. 42: 751-754.

Terasawa K, Toyota M, Sagae S, Ogi K, et al. (2006). Epigenetic inactivation of TCF2 in ovarian cancer and various cancer cell lines. Br. J. Cancer 94: 914-921.

Thomas G, Jacobs KB, Yeager M, Kraft P, et al. (2008). Multiple loci identified in a genome-wide association study of prostate cancer. Nat. Genet. 40: 310-315.

Tommasi S, Karm DL, Wu X, Yen Y, et al. (2009). Methylation of homeobox genes is a frequent and early epigenetic event in breast cancer. Breast Cancer Res. 11: R14.

Tronche F and Yaniv M (1992). HNF1, a homeoprotein member of the hepatic transcription regulatory network. Bioessays 14: 579-587.

Uemura H and Chang C (1998). Antisense TR3 orphan receptor can increase prostate cancer cell viability with etoposide treatment. Endocrinology 139: 2329-2334.

Wilhite SE and Barrett T (2012). Strategies to explore functional genomics data sets in NCBI's GEO database. Methods Mol. Biol. 802: 41-53.

Wixon J and Kell D (2000). The Kyoto Encyclopedia of Genes and Genomes - KEGG. Yeast 17: 48-55. 